\title{
ON INPUT-TO-OUTPUT STABILITY OF SWITCHED NON-EXPONENTIALLY STABLE NONLINEAR SYSTEMS
}

\author{
Denis Efimov Elena Panteley Antonio Loría
}

\begin{abstract}
The problem of input-to-output stability for switched nonlinear systems is considered. Two approaches for switching between non-exponentially stable systems are proposed guaranteeing input-to-output stability for the switched system.
\end{abstract}

\section{INTRODUCTION}

Stability and control theory of switched systems deals with generic problems involving the design of switching signals and establishing conditions on the latter to ensure stability. It is a well known fact that a switched system does not inherit the stability properties of the individual subsystems $-c f$. [6]. A commonly used assumption is to consider that switching among the subsystems is sufficiently slow or, in other words, that the switching signal has the so-called dwell time i.e., a minimal time-interval between two switches. In this case it may be shown, for a variety of stability concepts, that for a sufficiently large dwell-time constant, stability of the individual subsystems implies that of the switched system $-c f$. [3], [4], [5], [6], [7], [8], [9], [14], [15]. The constant dwell time assumption was weakened in [4], [14] by introducing average dwell time. In this case, it is required that a dwell-time interval between switches exist on sufficiently long intervals however, locally and only locally, it is possible to switch from one subsystem to another at any desired rate. In [10] state dependent dwell-time switching logic was introduced; this consists in making the length of time intervals between switches a function of the current system's state.

In [2] we showed that conventional dwell-time based switching preserves stability of (locally) exponentially stable systems. In this paper we extend previous results by proposing two methods for switched non-exponentially stable systems. The rest of the paper is organized as follows: in Section II we recall some definitions; our main theorems are formulated in Section III and an example is considered in Section IV. Concluding remarks are given in Section V.

D. Efimov is with the Dpt. of Electr. Engg and Comp. Sc., B28 Universite de Liège, Belgium; E. Panteley and A. Loría are with with C.N.R.S. at Laboratoire des Signaux et Systèmes, SUPELEC, 3, Rue Joliot Curie, 91192 Gif s/Yvette, France. E-mail: lorialiss. supelec.fr.

\section{PRELIMINARIES}

Consider a family of systems

$$
\dot{\mathbf{x}}=\mathbf{f}_{q}(\mathbf{x}, \mathbf{d}), \quad \mathbf{y}=\mathbf{h}(\mathbf{x}), \quad q \in I,
$$

where $\mathbf{x} \in R^{n}, \mathbf{d} \in R^{m}$ is a disturbance; $\mathbf{y} \in R^{p}$ is the output of interest and $q$ is an index in the (possibly unbounded) countable set $I$. We assume that $\mathbf{f}_{q}: R^{n+m} \rightarrow$ $R^{n}, q \in I, \mathbf{h}: R^{n} \rightarrow R^{p}$ are continuous and locally Lipschitz and that $\mathbf{d}: R_{+} \rightarrow R^{m}$ is Lebesgue measurable and essentially bounded:

$$
\|\mathbf{d}\|_{\left[t_{0}, t\right)}=e s s \sup \left\{|\mathbf{d}(t)|, t \in\left[t_{0}, t\right)\right\} .
$$

We denote by $M_{R^{m}}$ the set of globally essentially bounded functions, i.e., that satisfy $\|\mathbf{d}\|:=\|\mathbf{d}\|_{[0,+\infty)}<+\infty$.

A continuous function $\sigma: R_{+} \rightarrow R_{+}$is of class $K$ if it is strictly increasing and $\sigma(0)=0$; it is of class $K_{\infty}$ if it is also radially unbounded; a continuous function $\beta: R_{+} \times R_{+} \rightarrow R_{+}$is from class $\mathcal{K} \mathcal{L}$, if $\beta(\cdot, t) \in K$ for any $t \in R_{+}$, and $\beta(s, \cdot)$ is strictly decreasing to zero for each $s>0$.

Let $t_{0} \in R_{+}$and $i: R_{\geq t_{0}} \rightarrow I$ be piecewise constant and right-continuous, then, for $q=i(t)$ the family of systems (1) defines the following switched system

$$
\dot{\mathbf{x}}=\mathbf{f}_{i(t)}(\mathbf{x}, \mathbf{d}), \quad \mathbf{y}=\mathbf{h}(\mathbf{x}) .
$$

Following [4], [7], [10], [14] we recall the definition of dwell-time.

Definition 1. The switching signal $i(t)$ is said to have average dwell-time $0<\tau_{D}<+\infty$, if there exists an integer $N_{0}$ such that $1 \leq N_{0}<+\infty$ and, for any $t_{2} \geq t_{1} \geq 0$ we have

$$
N_{\left[t_{1}, t_{2}\right)} \leq N_{0}+\frac{t_{2}-t_{1}}{\tau_{D}},
$$

where $N_{\left[t_{1}, t_{2}\right)}$ is the number of switches. If the interval between any two switches is not less than $\tau_{D}$ the switching signal $i(t)$ has dwell-time and $N_{0}=1$.

The system (2), where $i(t)$ has average dwell-time or constant dwell-time, has finite number of switches on any finite-time interval and its solution is continuous and defined, at least, locally.

The switched system (2) is called forward complete if, for all initial conditions $t_{0} \geq 0, \mathbf{x}_{0} \in R^{n}$ and all inputs $\mathbf{d} \in M_{R^{m}}$, the solutions $\mathbf{x}\left(t, t_{0}, \mathbf{x}_{0}, \mathbf{d}\right)$ of the switched system (2) are defined for all $t \geq t_{0}$ (necessary and sufficient conditions for a dynamical system (1) with fixed 
$q$ to be forward complete can be found in [1]). We denote the output trajectory $\mathbf{y}\left(t, t_{0}, \mathbf{x}_{0}, \mathbf{d}\right)=\mathbf{h}\left(\mathbf{x}\left(t, t_{0}, \mathbf{x}_{0}, \mathbf{d}\right)\right)$ and, on occasions, we use the short-hand notations $\mathbf{x}(t)=$ $\mathbf{x}\left(t, t_{0}, \mathbf{x}_{0}, \mathbf{d}\right), \mathbf{y}(t)=\mathbf{y}\left(t, t_{0}, \mathbf{x}_{0}, \mathbf{d}\right)$. Furthermore, we recall the following stability definitions from [12], [13].

Definition 2. We say that for some fixed $q \in I$ a forward complete system (1) is Input-to-Output Stable (IOS) with respect to the output $\mathbf{y}$ and the input $\mathbf{d}$ if there exist functions $\beta_{q} \in \mathcal{K} \mathcal{L}$ and $\gamma_{q} \in K$ such that for all $t_{0} \in$ $R_{+}, \mathbf{x}_{0} \in R^{n}, \mathbf{d} \in M_{R^{m}}$, and all $t \geq t_{0}$

$$
\left|\mathbf{y}\left(t, t_{0}, \mathbf{x}_{0}, \mathbf{d}\right)\right| \leq \beta_{q}\left(\left|\mathbf{x}_{0}\right|, t-t_{0}\right)+\gamma_{q}\left(\|\mathbf{d}\|_{\left[t_{0}, t\right)}\right) .
$$

We say that a switched forward complete system (2) with $i: R_{\geq t_{0}} \rightarrow I$ is IOS with respect to the output $\mathbf{y}$ and the input $\mathbf{d}$ if there exist functions $\beta \in \mathcal{K} \mathcal{L}$ and $\gamma \in K$ such that, for all $t_{0} \in R_{+}, \mathbf{x}_{0} \in R^{n}, \mathbf{d} \in M_{R^{m}}$, and all $t \geq t_{0}$,

$$
\left|\mathbf{y}\left(t, t_{0}, \mathbf{x}_{0}, \mathbf{d}\right)\right| \leq \beta\left(\left|\mathbf{x}_{0}\right|, t-t_{0}\right)+\gamma\left(\|\mathbf{d}\|_{\left[t_{0}, t\right)}\right),
$$

Definition 3. We say that for some fixed $q \in I$ the forward complete system (1) is state independent IOS (SIIOS) with respect to the output $\mathbf{y}$ and the input $\mathbf{d}$ if there exist functions $\beta_{q}^{\prime} \in \mathcal{K} \mathcal{L}$ and $\gamma_{q}^{\prime} \in K$ such that, for all $t_{0} \in R_{+}, \mathbf{x}_{0} \in R^{n}, \mathbf{d} \in M_{R^{m}}$, and all $t \geq t_{0}$,

$$
\left|\mathbf{y}\left(t, t_{0}, \mathbf{x}_{0}, \mathbf{d}\right)\right| \leq \beta_{q}^{\prime}\left(\left|\mathbf{h}\left(\mathbf{x}_{0}\right)\right|, t-t_{0}\right)+\gamma_{q}^{\prime}\left(\|\mathbf{d}\|_{\left[t_{0}, t\right)}\right) .
$$

We say that a switched forward complete system (2) with $i: R_{\geq t_{0}} \rightarrow I$ is SIIOS with respect to the output $\mathbf{y}$ and the input $\mathbf{d}$ if there exist functions $\beta^{\prime} \in \mathcal{K} \mathcal{L}$ and $\gamma^{\prime} \in K$ such that, for all $t_{0} \in R_{+}, \mathbf{x}_{0} \in R^{n}, \mathbf{d} \in M_{R^{m}}$, and all $t \geq t_{0}$

$\left|\mathbf{y}\left(t, t_{0}, \mathbf{x}_{0}, \mathbf{d}\right)\right| \leq \beta^{\prime}\left(\left|\mathbf{h}\left(\mathbf{x}_{0}\right)\right|, t-t_{0}\right)+\gamma^{\prime}\left(\|\mathbf{d}\|_{\left[t_{0}, t\right)}\right)$.

The systems are exponentially SIIOS if $\beta_{q}^{\prime}(s, r)=$ $a s e^{-b r}$ or $\beta^{\prime}(s, r)=a s e^{-b r}$ for some $a>0, b>0$.

The difference between IOS and SIIOS consists in the dependence on initial conditions. For SIIOS system if initial the amplitude of the variable $\mathbf{y}$ is small, then the overall amplitude of $\mathbf{y}$ during the transient is also "small". For an IOS system the output $\mathbf{y}$,even with small initial values, may have large transient values if $\mathbf{x}_{0}$ is "large" in norm. In contrast to this, the asymptotic Al behavior of IOS and SIIOS systems' trajectories is similar: in either case, and in the absence of disturbances, the trajectories converge to the set $\{\mathbf{h}(\mathbf{x})=0\}$. In the particular case when $\mathbf{y}=\mathbf{x}$ both properties boil down to the well known input-to-state stability (ISS) property [11].

In the sequel we make the following standing assumption.

Assumption 1. For each fixed $q \in I$ system from (1) is forward complete and SIIOS with respect to output $\mathbf{y}$ and the input $\mathbf{d}$ for functions $\beta_{q} \in \mathcal{K} \mathcal{L}$ and $\gamma \in K$.

Note that from Definition 3 it holds that $\beta_{q}(s, 0) \geq s$, $s \geq 0$.

\section{MAIN RESULTS}

In [15] it is shown under suitable smoothness assumptions on the switched systems that if Assumption 1 holds for $\mathbf{y}=\mathbf{x}$ and the switching signal has dwell-time $\tau_{D}>0$ for all $j \in I$ and $s \geq 0$

$$
\beta_{j}\left(s, \tau_{D}\right) \leq \lambda s, \quad 0<\lambda<1
$$

the switched system (2) is SIIOS. In [14] an extension of this result for the case of average dwell-time is presented and to the more general case of $\mathbf{h}(\mathbf{x})$ in [2]. In the latter we also show that (3) is equivalent to exponential stability of all subsystems in (1). An attempt to overcome the condition on exponential stability was made in [10], where authors introduced a state-dependent variant of dwell time. A key property to ensure existence is local Lipschitz continuity of functions $\beta_{j}$ with respect to the first argument near zero, that implies local exponential stability for all subsystems in (1) -see [2] for details.

To weaken the exponential stability condition (3) for systems satisfying Assumption 1, under constant or average dwell time, leads to practical asymptotic stability of the switched system $-c f$. [10]. That is, there exists $\varepsilon>0$ and functions $\beta^{\prime} \in \mathcal{K} \mathcal{L}, \gamma^{\prime} \in K$ such that, for all $t_{0} \in R_{+}$, $\mathbf{x}_{0} \in R^{n}$ and $\mathbf{d} \in M_{R^{m}}$,

$\left|\mathbf{y}\left(t, t_{0}, \mathbf{x}_{0}, \mathbf{d}\right)\right| \leq \beta^{\prime}\left(\left|\mathbf{h}\left(\mathbf{x}_{0}\right)\right|, t-t_{0}\right)+\gamma^{\prime}\left(\|\mathbf{d}\|_{\left[t_{0}, t\right)}\right)+\varepsilon$.

The technical reason which leads to conclude practical as opposite to asymptotic Al stability of $\{\mathbf{y}=0\}$ in [10], is that the case when a finite number of switches occurs, is excluded. Indeed, if we rule out switches in a neighborhood of $\{\mathbf{y}=0\}$ then it is possible to conclude SIIOS even for non-exponentially stable systems (1). Below, we present alternative conditions to establish exponential SIIOS.

\section{A. Partial exponential stability of the family (1)}

The first solution deals with the case when Assumption 1 fails only for some of subsystems in (1). That is, let us assume that $I=I_{1} \cup I_{2}$ where the systems corresponding to values of $q \in I_{1}$ are not exponentially stable and those corresponding to values of $q \in I_{2}$ are exponential SIIOS stable.

Theorem 1. Let Assumption 1 hold, $I=I_{1} \cup I_{2}$ and there exist $0<\tau_{D}<+\infty$ and $\kappa>1$ such that

$$
\begin{gathered}
\bar{\beta}_{2}\left(2 \bar{\beta}_{1}(s), \tau_{D}\right) \leq \lambda s, \quad s \geq 0, \quad 0<\lambda<1, \\
\bar{\beta}_{1}(s)=\sup _{j_{1}, \ldots, j_{\kappa} \in I_{1}}\left\{\beta_{j_{1}}\left(\ldots 2 \beta_{j_{\kappa}}(s, 0) \ldots, 0\right)\right\}, \quad s \geq 0 ; \\
\bar{\beta}_{2}(s, t)=\sup _{j \in I_{2}}\left\{\beta_{j}(s, t)\right\}, \quad s \geq 0, \quad t \geq 0 .
\end{gathered}
$$

Let the switching signal $i(t)$ satisfy the following conditions for any $j \geq 0\left(t_{j}\right.$ are switching instants $)$ :

$$
\begin{gathered}
i(t) \in I_{1}, \quad t \in\left[t_{j}, t_{j+1}\right) \quad \Rightarrow \quad t_{j+1}-t_{j}>0 \\
i(t) \in I_{2}, \quad t \in\left[t_{j}, t_{j+1}\right) \quad \Rightarrow \quad t_{j+1}-t_{j} \geq \tau_{D}
\end{gathered}
$$


for all $j \geq 0$ there exists $0 \leq j^{\prime} \leq \kappa$ such that

$$
\left\{i\left(t_{j}\right) \in I_{2} \quad \Rightarrow i\left(t_{j+j^{\prime}}\right) \in I_{2}\right\} .
$$

Then, for all $t_{0} \in R_{+}, \mathbf{x}_{0} \in R^{n}, \mathbf{d} \in M_{R^{m}}$ and all $t \geq t_{0}$,

$$
\begin{gathered}
|\mathbf{y}(t)| \leq \bar{\beta}_{1}\left(2 \bar{\beta}_{2}\left(2 \bar{\beta}_{1}\left(\left|\mathbf{y}\left(t_{0}\right)\right|\right), 0\right)\right)+\bar{\beta}_{1}(2 \gamma(\|\mathbf{d}\|))+\gamma(\|\mathbf{d}\|), \\
\limsup _{t \rightarrow+\infty}|\mathbf{y}(t)| \leq \bar{\beta}_{1}(2 \bar{\gamma}(\|\mathbf{d}\|))+\gamma(\|\mathbf{d}\|) .
\end{gathered}
$$

where

$$
\bar{\gamma}(s)=\bar{\beta}_{2}\left(4 \Lambda \kappa \bar{\beta}_{1}(\gamma(s)), 0\right)+\gamma(s), \quad \Lambda=1 /(1-\lambda)
$$

Proof. Properties (5)-(7) imply that, when $i(t) \in I_{1}$ the switches with any desired frequency, are possible and dwell-time $\tau_{D}$ affects only the time that systems (2) such that $i(t) \in I_{2}$ are active. According to (7) the system has a property similar to average dwell-time with $\tau_{D} / \kappa$ and $N_{0}=\kappa$ however, it is not average dwell-time in the sense of Definition 1 since systems from $i(t) \in I_{2}$ cannot be active on time intervals with length smaller than $\tau_{D}$ and the frequency with which these systems become active is bounded from below by $\kappa$. The solutions of system (2) are continuous and defined, at least locally, on $\left[t_{0}, T\right)$. Actually, $T=+\infty$ since for each fixed $t$ the solutions of system (2) equal those of a system from family (1) for some fixed $q \in I$, which is forward complete by assumption.

Consider any time interval $\left[t_{0}, T\right), T \geq t_{0}$. Without loosing generality the interval can be presented as concatenation of subintervals:

$$
\left[t_{0}, T\right)=\bigcup_{j=0}^{k}\left[t_{j}, t_{j+1}\right), \quad t_{k+1}=T
$$

with the property that for each $j \geq 0$ there exists $t_{j}^{\prime} \in$ $\left(t_{j}, t_{j+1}\right)$ such that $i(t) \in I_{1}$ for $t \in\left[t_{j}, t_{j}^{\prime}\right)$ and $i(t) \in I_{2}$ for $t \in\left[t_{j}^{\prime}, t_{j+1}\right)$ and there are no switches in the latter subinterval. From Assumption 1 (from (7) on the interval $\left[t_{j}, t_{j}^{\prime}\right)$ only $\kappa$ switches are possible as a maximum) we have

$$
\begin{aligned}
& |\mathbf{y}(t)| \leq \bar{\beta}_{1}\left(\left|\mathbf{y}\left(t_{j}\right)\right|\right)+\kappa \bar{\beta}_{1}\left(\gamma\left(\|\mathbf{d}\|_{\left[t_{j}, t_{j}^{\prime}\right)}\right)\right), \quad t \in\left[t_{j}, t_{j}^{\prime}\right) ; \\
& |\mathbf{y}(t)| \leq \bar{\beta}_{2}\left(\left|\mathbf{y}\left(t_{j}^{\prime}\right)\right|, t-t_{j}^{\prime}\right)+\gamma\left(\|\mathbf{d}\|_{\left[t_{j}^{\prime}, t_{j+1}\right)}\right), t \in\left[t_{j}^{\prime}, t_{j+1}\right)
\end{aligned}
$$

then, using (4) and $t_{j+1}-t_{j}^{\prime} \geq \tau_{D}$ (the latter holds due to (6)) we obtain

$$
\begin{aligned}
& \left|\mathbf{y}\left(t_{j+1}\right)\right| \leq \\
& \bar{\beta}_{2}\left(\bar{\beta}_{1}\left(\left|\mathbf{y}\left(t_{j}\right)\right|\right)+\kappa \bar{\beta}_{1}\left(\gamma\left(\|\mathbf{d}\|_{\left[t_{j}, t_{j}^{\prime}\right)}\right)\right), t_{j+1}-t_{j}^{\prime}\right) \\
& \quad+\gamma\left(\|\mathbf{d}\|_{\left[t_{j}^{\prime}, t_{j+1}\right)}\right) \\
& \leq \beta_{2}\left(2 \bar{\beta}_{1}\left(\left|\mathbf{y}\left(t_{j}\right)\right|\right), t_{j+1}-t_{j}^{\prime}\right) \\
& \quad+\beta_{2}\left(2 \kappa \bar{\beta}_{1}\left(\gamma\left(\|\mathbf{d}\|_{\left[t_{j}, t_{j}^{\prime}\right)}\right)\right), t_{j+1}-t_{j}^{\prime}\right) \\
& \quad+\gamma\left(\mathbf{d} \|_{\left[t_{j}^{\prime}, t_{j+1}\right)}\right) \\
& \leq \lambda\left|\mathbf{y}\left(t_{j}\right)\right|+\lambda \kappa \bar{\beta}_{1}\left(\gamma\left(\|\mathbf{d}\|_{\left[t_{j}, t_{j}^{\prime}\right)}\right)\right)+\gamma\left(\|\mathbf{d}\|_{\left[t_{j}^{\prime}, t_{j+1}\right)}\right) \\
& \leq \lambda\left|\mathbf{y}\left(t_{j}\right)\right|+(\lambda+1) \kappa \bar{\beta}_{1}\left(\gamma\left(\|\mathbf{d}\|_{\left[t_{j}, t_{j+1}\right)}\right)\right)
\end{aligned}
$$

and for all $t \in\left(t_{j}, t_{j+1}\right)$

$$
\begin{aligned}
& |\mathbf{y}(t)| \leq \beta_{2}\left(2 \bar{\beta}_{1}\left(\left|\mathbf{y}\left(t_{j}\right)\right|\right), 0\right) \\
& \quad+\beta_{2}\left(2 \kappa \bar{\beta}_{1}\left(\gamma\left(\|\mathbf{d}\|_{\left[t_{j}, t_{j}^{\prime}\right)}\right)\right), 0\right)+\gamma\left(\|\mathbf{d}\|_{\left[t_{j}^{\prime}, t_{j+1}\right)}\right) .
\end{aligned}
$$

Applying this procedure for all $0 \leq j \leq k$ we obtain:

$$
\begin{aligned}
& \left|\mathbf{y}\left(t_{k+1}\right)\right| \leq \\
& \lambda\left[\lambda \cdots\left[\lambda\left|\mathbf{y}\left(t_{0}\right)\right|+(\lambda+1) \kappa \bar{\beta}_{1}\left(\gamma\left(\|\mathbf{d}\|_{\left[t_{0}, t_{1}\right)}\right)\right)\right] \cdots\right. \\
& \left.+(\lambda+1) \kappa \bar{\beta}_{1}\left(\gamma\left(\|\mathbf{d}\|_{\left[t_{k-1}, t_{k}\right.}\right)\right)\right] \\
& +(\lambda+1) \kappa \bar{\beta}_{1}\left(\gamma\left(\|\mathbf{d}\|_{\left[t_{k}, t_{k+1}\right)}\right)\right) \\
& \leq \lambda^{k}\left|\mathbf{y}\left(t_{0}\right)\right|+(\lambda+1) \sum_{j=0}^{k} \lambda^{j} \kappa \bar{\beta}_{1}\left(\gamma\left(\|\mathbf{d}\|_{\left[t_{k-j}, t_{k-j+1}\right)}\right)\right) \\
& \leq \lambda^{k}\left|\mathbf{y}\left(t_{0}\right)\right|+2 \Lambda \kappa \bar{\beta}_{1}\left(\gamma\left(\|\mathbf{d}\|_{\left[t_{0}, t_{k+1}\right)}\right)\right) .
\end{aligned}
$$

If there exists infinite number of switches in the system $(k \rightarrow+\infty)$, then:

$$
\begin{gathered}
|\mathbf{y}(t)| \leq \bar{\beta}_{2}\left(2 \bar{\beta}_{1}\left(\left|\mathbf{y}\left(t_{0}\right)\right|\right), 0\right)+\bar{\gamma}(\|\mathbf{d}\|), \quad t \geq t_{0}, \\
\limsup _{t \rightarrow+\infty}|\mathbf{y}(t)| \leq \bar{\gamma}(\|\mathbf{d}\|) .
\end{gathered}
$$

If the number of switches is finite and $k \leq K<+\infty$, then for the case $i\left(t_{K}\right) \in I_{2}$ the same estimates are satisfied, while for $i\left(t_{K}\right) \in I_{1}$ we have, for all $t \geq t_{0}$

$$
\begin{gathered}
|\mathbf{y}(t)| \leq \bar{\beta}_{1}\left(2 \bar{\beta}_{2}\left(2 \bar{\beta}_{1}\left(\left|\mathbf{y}\left(t_{0}\right)\right|\right), 0\right)\right)+\bar{\beta}_{1}(2 \bar{\gamma}(\|\mathbf{d}\|))+\gamma(\|\mathbf{d}\|), \\
\limsup _{t \rightarrow+\infty}|\mathbf{y}(t)| \leq \bar{\beta}_{1}(2 \bar{\gamma}(\|\mathbf{d}\|))+\gamma(\|\mathbf{d}\|) .
\end{gathered}
$$

Combining these estimates the result follows.

Theorem 1 is stated in terms of a switching rule which is less conservative than constant-dwell-time based algorithms since the systems from subset $I_{1}$ can be active on any sufficiently small time interval. However, such new switching rule is more restrictive than average dwell-time in the sense that the systems from $I_{2}$ must remain active during the time intervals with length bigger than $\tau_{D}$ and with frequency $\kappa$.

\section{B. Non-uniform convergence under dwell-time}

Under Assumption 1 exponential SIIOS stability of systems (1) follows from (3) provided that output trajectories decrease (in norm) by a factor of $\lambda$ over intervals of length larger or equal to the dwell time. In non-exponentially stable systems the length of such intervals may be decreased as a function of the output norm. In this case, the condition (3) can be relaxed to the following.

Assumption 2. For any given $S>0$ and $\tau_{D}>0$ there exists a continuous function $\rho:(0, S) \rightarrow(0,1)$ such that for all $0<s<S$ and $j \in I$

$$
\beta_{j}\left(s, \tau_{D}\right) \leq \rho(s) s \text {. }
$$

Assumption 2 means that on any interval longer than the dwell time $\tau_{D}$ the output trajectory of (1) for any $q$ decreases in norm by a factor of $\rho\left(\left|\mathbf{x}_{0}\right|\right) \leq 1$. we claim 
that Assumption 2 holds if $\beta_{j}(s, 0)=s, 0 \leq s \leq S, j \in I$. To see this, take $\sup _{j \in I} \frac{\beta_{j}\left(s, \tau_{D}\right)}{s} \leq \rho(s) \leq \sup _{j \in I} \frac{\beta_{j}(s, 0)}{s} \leq 1$. Clearly, $\rho \geq 0$ and it is strictly positive except for the case when there exists $s^{\prime} \in(0, S)$ such that

$$
\max _{j \in I} \frac{\beta_{j}\left(s^{\prime}, 0\right)}{s^{\prime}}=0 .
$$

However, the latter cannot hold for any $s^{\prime} \in(0, S)$ and $\tau_{D}>0$ due to the properties of $\mathcal{K} \mathcal{L}$ functions. By the same arguments $\beta_{j}\left(s, \tau_{D}\right)<s$ for $s \in(0, S)$ and $\rho$ can be chosen to be smaller than 1 on this interval. Indeed, if $\rho_{\max }=\sup _{0<s<S} \rho(s)<1$ then each system in (1) is locally exponentially stable for $s \in(0, S)$. In the sequel, we focus on situations where this property fails.

Firstly, let us consider output asymptotic stability of switched systems without disturbances.

Theorem 2. Let Assumptions 1 and 2 hold for a given $R>$ $0, S=S(R)=\bar{\beta}(R), \tau_{D}>0$, and $\bar{\beta}(s)=\max _{j \in I}\left\{\beta_{j}(s, 0)\right\}$. Then, for all $t_{0} \in R_{+}, \mathbf{x}_{0} \in X_{R}, X_{R} \stackrel{j \in I}{=}\left\{\mathbf{x} \in R^{n}\right.$ : $|\mathbf{h}(\mathbf{x})|<R\}$ and $\|\mathbf{d}\|=0$ the solutions of the switched system (2) satisfy

$$
|\mathbf{y}(t)| \leq \bar{\beta}\left(\left|\mathbf{h}\left(\mathbf{x}_{0}\right)\right|\right), \quad \forall t \geq t_{0} ; \quad \limsup _{t \rightarrow+\infty}|\mathbf{y}(t)|=0
$$

provided that signal $i(t)$ has dwell time $\tau_{D}$.

Proof. Since Assumption 1 holds and the switching signal has dwell-time, the solutions of (2) are continuous and defined for all $t \geq 0$. For the time being let us assume that there exists an infinite series of switches $\left\{t_{i}\right\}, i=0,1, \ldots$ with the property that $t_{i+1}-t_{i} \geq \tau_{D}$ for all $i \geq 0$. Then, on the first interval where the switching signal remains constant i.e., for all $t \in\left[t_{0}, t_{1}\right)$, we have

$$
|\mathbf{y}(t)| \leq \beta_{i\left(t_{0}\right)}\left(\left|\mathbf{h}\left(\mathbf{x}_{0}\right)\right|, t-t_{0}\right) \leq \beta_{i\left(t_{0}\right)}\left(\left|\mathbf{h}\left(\mathbf{x}_{0}\right)\right|, 0\right) .
$$

Due to Assumption 2 and since $\left|\mathbf{y}\left(t_{0}\right)\right|<S$ we have $\left|\mathbf{y}\left(t_{1}\right)\right| \leq \rho_{0}\left|\mathbf{h}\left(\mathbf{x}_{0}\right)\right|$ where $\rho_{i}=\rho\left(\left|\mathbf{h}\left(\mathbf{x}\left(t_{i}\right)\right)\right|\right)$ and $i \geq 0$. Over the next interval $t \in\left[t_{1}, t_{2}\right)$ we have

$$
\begin{aligned}
|\mathbf{y}(t)| & \leq \beta_{i\left(t_{1}\right)}\left(\left|\mathbf{h}\left(\mathbf{x}\left(t_{1}\right)\right)\right|, t-t_{1}\right) \\
& \leq \beta_{i\left(t_{1}\right)}\left(\rho_{0}\left|\mathbf{h}\left(\mathbf{x}_{0}\right)\right|, 0\right) \\
\left|\mathbf{y}\left(t_{2}\right)\right| & \leq \rho_{1} \rho_{0}\left|\mathbf{h}\left(\mathbf{x}_{0}\right)\right|
\end{aligned}
$$

Applying similar arguments for any arbitrary interval $\left[t_{i}, t_{i+1}\right)$ with $i>0$, we obtain

$$
\begin{aligned}
|\mathbf{y}(t)| & \leq \beta_{i\left(t_{i}\right)}\left(\left|\mathbf{h}\left(\mathbf{x}\left(t_{i}\right)\right)\right|, t-t_{i}\right) \\
& \leq \beta_{i\left(t_{i}\right)}\left(\rho_{i-1} \ldots \rho_{0}\left|\mathbf{h}\left(\mathbf{x}_{0}\right)\right|, 0\right), \\
\left|\mathbf{y}\left(t_{i+1}\right)\right| & \leq \rho_{i} \ldots \rho_{0}\left|\mathbf{h}\left(\mathbf{x}_{0}\right)\right| .
\end{aligned}
$$

The latter estimates imply output uniform boundedness and attractivity for all $0<\rho_{i}<1, i \geq 0$ such that $0<\left|\mathbf{y}\left(t_{i}\right)\right|<S$ which holds in view of the choice of $S=S(R)$. Combining these estimates and the estimates derived for the case $i=0$ we obtain

$$
|\mathbf{y}(t)| \leq \bar{\beta}\left[\left|\mathbf{h}\left(\mathbf{x}_{0}\right)\right|\right], \quad \forall t \geq t_{0} ; \quad \limsup _{t \rightarrow+\infty}|\mathbf{y}(t)|=0 .
$$

The latter is also valid if the number of switches is finite.

Now, we consider the case with disturbances.

Theorem 3. Let Assumption 1 and Assumption 2 hold (with $S=+\infty, \tau_{D}>0$ ) and let the disturbances satisfy $\int_{0}^{+\infty} \gamma(|\mathbf{d}(t)|) d t<+\infty$. Then, for all $t_{0} \in R_{+}, \mathbf{x}_{0} \in R^{n}$ and $\mathbf{d} \in M_{R^{m}}$ the solutions of the switched system (2) admit the following estimates for some $0<c<+\infty$ and for all $t \geq t_{0}$

$$
\begin{gathered}
|\mathbf{y}(t)| \leq \bar{\beta}\left(2\left|\mathbf{h}\left(\mathbf{x}_{0}\right)\right|\right)+\bar{\beta}(2 c \gamma(\|\mathbf{d}\|)+2)+\gamma(\|\mathbf{d}\|), \\
\limsup _{t \rightarrow+\infty}|\mathbf{y}(t)|=0 .
\end{gathered}
$$

Proof. From Assumption 1 and the dwell-time property on the switching signal we obtain the existence of solutions of (2) for all $t \geq t_{0}$. Consider the series of switches $\left\{t_{i}\right\}$, where $i=0,1, \ldots+\infty$ with the property $t_{i+1}-t_{i} \geq \tau_{D}$, $i \geq 0$. Then, over the first interval $\left[t_{0}, t_{1}\right)$ we have

$$
\begin{aligned}
|\mathbf{y}(t)| & \leq \beta_{i\left(t_{0}\right)}\left(\left|\mathbf{h}\left(\mathbf{x}_{0}\right)\right|, t-t_{0}\right)+\gamma\left(\|\mathbf{d}\|_{\left[t_{0}, t\right)}\right) \\
& \leq \beta_{i\left(t_{0}\right)}\left(\left|\mathbf{h}\left(\mathbf{x}_{0}\right)\right|, 0\right)+\gamma\left(\|\mathbf{d}\|_{\left[t_{0}, t\right)}\right) .
\end{aligned}
$$

Since $S=+\infty$ then, due to Assumption 2 (denote $\rho_{i}=$ $\left.\rho\left(\left|\mathbf{h}\left(\mathbf{x}\left(t_{i}\right)\right)\right|\right), i \geq 0\right)$

$$
\left|\mathbf{y}\left(t_{1}\right)\right| \leq \rho_{0}\left|\mathbf{h}\left(\mathbf{x}_{0}\right)\right|+\gamma\left(\|\mathbf{d}\|_{\left[t_{0}, t_{1}\right)}\right) .
$$

Similarly, on the next interval i.e., for all $t \in\left[t_{1}, t_{2}\right)$,

$$
\begin{aligned}
& |\mathbf{y}(t)| \leq \beta_{i\left(t_{1}\right)}\left(\left|\mathbf{h}\left(\mathbf{x}\left(t_{1}\right)\right)\right|, t-t_{1}\right)+\gamma\left(\|\mathbf{d}\|_{\left[t_{1}, t\right)}\right) \\
& \quad \leq \beta_{i\left(t_{1}\right)}\left(\rho_{0}\left|\mathbf{h}\left(\mathbf{x}_{0}\right)\right|+\gamma\left(\|\mathbf{d}\|_{\left[t_{0}, t_{1}\right)}\right), 0\right)+\gamma\left(\|\mathbf{d}\|_{\left[t_{1}, t\right)}\right), \\
& \left|\mathbf{y}\left(t_{2}\right)\right| \leq \rho_{1} \rho_{0}\left|\mathbf{h}\left(\mathbf{x}_{0}\right)\right|+\rho_{1} \gamma\left(\|\mathbf{d}\|_{\left[t_{0}, t_{1}\right)}\right)+\gamma\left(\|\mathbf{d}\|_{\left[t_{1}, t_{2}\right)}\right) .
\end{aligned}
$$

And for all $t \in\left[t_{2}, t_{3}\right)$ :

$$
\begin{array}{r}
|\mathbf{y}(t)| \leq \beta_{i\left(t_{2}\right)}\left(\left|\mathbf{h}\left(\mathbf{x}\left(t_{2}\right)\right)\right|, t-t_{2}\right)+\gamma\left(\|\mathbf{d}\|_{\left[t_{2}, t\right)}\right) \\
\leq \beta_{i\left(t_{2}\right)}\left(\rho_{1} \rho_{0}\left|\mathbf{h}\left(\mathbf{x}_{0}\right)\right|+\rho_{1} \gamma\left(\|\mathbf{d}\|_{\left[t_{0}, t_{1}\right)}\right)\right. \\
\left.+\gamma\left(\|\mathbf{d}\|_{\left[t_{1}, t_{2}\right)}\right), 0\right)+\gamma\left(\|\mathbf{d}\|_{\left[t_{2}, t\right)}\right) \\
\left|\mathbf{y}\left(t_{3}\right)\right| \leq \quad \rho_{2} \rho_{1} \rho_{0}\left|\mathbf{h}\left(\mathbf{x}_{0}\right)\right|+\rho_{2} \rho_{1} \gamma\left(\|\mathbf{d}\|_{\left[t_{0}, t_{1}\right)}\right) \\
+\rho_{2} \gamma\left(\|\mathbf{d}\|_{\left[t_{1}, t_{2}\right)}\right)+\gamma\left(\|\mathbf{d}\|_{\left[t_{2}, t_{3}\right)}\right)
\end{array}
$$

Applying similar arguments for any arbitrary interval we obtain, for all $t \in\left[t_{i}, t_{i+1}\right), i>0$ :

$$
\begin{aligned}
& |\mathbf{y}(t)| \leq \beta_{i\left(t_{i}\right)}\left(\left|\mathbf{h}\left(\mathbf{x}\left(t_{i}\right)\right)\right|, t-t_{i}\right)+\gamma\left(\|\mathbf{d}\|_{\left[t_{i}, t\right)}\right) \\
& \leq \beta_{i\left(t_{i}\right)}\left(\rho_{i-1} \cdots \rho_{0}\left|\mathbf{h}\left(\mathbf{x}_{0}\right)\right|\right. \\
& \left.\quad+\sum_{j=0}^{i-1}\left(\prod_{k=j+1}^{i-1} \rho_{k}\right) \gamma\left(\|\mathbf{d}\|_{\left[t_{j}, t_{j+1}\right)}\right), 0\right) \\
& \quad+\gamma\left(\|\mathbf{d}\|_{\left[t_{i}, t\right)}\right),
\end{aligned}
$$




$$
\begin{aligned}
\left|\mathbf{y}\left(t_{i+1}\right)\right| \leq \rho_{i} \ldots \rho_{0}\left|\mathbf{h}\left(\mathbf{x}_{0}\right)\right|+\gamma\left(\|\mathbf{d}\|_{\left[t_{i}, t\right)}\right) & \\
& +\sum_{j=0}^{i-1}\left(\prod_{k=j+1}^{i} \rho_{k}\right) \gamma\left(\|\mathbf{d}\|_{\left[t_{j}, t_{j+1}\right)}\right)
\end{aligned}
$$

where it is supposed that $\prod_{k=k_{1}}^{k_{2}} \rho_{k}=1$ if $k_{1}>k_{2}$. Combining these estimates and the estimates derived for the case $i=0$ we obtain

$$
\begin{array}{r}
|\mathbf{y}(t)| \leq \bar{\beta}\left(2\left|\mathbf{h}\left(\mathbf{x}_{0}\right)\right|\right)+\gamma\left(\|\mathbf{d}\|_{\left[t_{0}, t\right)}\right), \\
+\bar{\beta}\left(2 \sum_{j=0}^{i-1}\left(\prod_{k=j+1}^{i-1} \rho_{k}\right) \gamma\left(\|\mathbf{d}\|_{\left[t_{0}, t\right)}\right)\right) \\
\limsup _{t \rightarrow+\infty}|\mathbf{y}(t)| \leq \sum_{j=0}^{i-1}\left(\prod_{k=j+1}^{i} \rho_{k}\right) \gamma(\|\mathbf{d}\|)+\gamma(\|\mathbf{d}\|) .
\end{array}
$$

Note, that these estimates are also valid for the case of finite number of switches. Let us stress that without additional assumptions the term proportional to the norm of disturbance can be unbounded since $0<\rho_{k}<1$. Furthermore, for any $\varepsilon>0$ there exists $i_{\varepsilon} \geq 0$ such that

$$
\sum_{j=i_{\varepsilon}}^{\infty} \gamma\left(\|\mathbf{d}\|_{\left[t_{j}, t_{j+1}\right)}\right) \leq \varepsilon
$$

In this case we have for all $t \in\left[t_{i}, t_{i+1}\right)$ and $i>0$,

$$
\begin{aligned}
&|\mathbf{y}(t)| \leq \bar{\beta}\left(2\left|\mathbf{h}\left(\mathbf{x}_{0}\right)\right|\right)+\gamma\left(\|\mathbf{d}\|_{\left[t_{i}, t\right)}\right), \\
&+\bar{\beta}\left(2 \sum_{j=0}^{i-1}\left(\prod_{k=j+1}^{i-1} \rho_{k}\right) \gamma\left(\|\mathbf{d}\|_{\left[t_{j}, t_{j+1}\right)}\right)\right) \\
&\left|\mathbf{y}\left(t_{i+1}\right)\right| \leq \rho_{i} \ldots \rho_{0}\left|\mathbf{h}\left(\mathbf{x}_{0}\right)\right|+\gamma\left(\|\mathbf{d}\|_{\left[t_{i}, t\right)}\right) \\
&+\sum_{j=0}^{i-1}\left(\prod_{k=j+1}^{i} \rho_{k}\right) \gamma\left(\|\mathbf{d}\|_{\left[t_{j}, t_{j+1}\right)}\right)
\end{aligned}
$$

and

$$
\begin{aligned}
& \sum_{j=0}^{\infty}\left(\prod_{k=j+1}^{\infty} \rho_{k}\right) \gamma\left(\|\mathbf{d}\|_{\left[t_{j}, t_{j+1}\right)}\right) \\
& \quad \leq i_{\varepsilon} \gamma(\|\mathbf{d}\|)+\sum_{j=i_{\varepsilon}}^{\infty}\left(\prod_{k=j+1}^{\infty} \rho_{k}\right) \gamma\left(\|\mathbf{d}\|_{\left[t_{j}, t_{j+1}\right)}\right) \\
& \quad \leq i_{\varepsilon} \gamma(\|\mathbf{d}\|)+\sum_{j=i_{\varepsilon}}^{\infty} \gamma\left(\|\mathbf{d}\|_{\left[t_{j}, t_{j+1}\right)}\right) \leq i_{\varepsilon} \gamma(\|\mathbf{d}\|)+\varepsilon .
\end{aligned}
$$

Therefore, for each $\varepsilon>0$ and for all $t \geq t_{0}$,

$$
\begin{gathered}
|\mathbf{y}(t)| \leq \bar{\beta}\left(2\left|\mathbf{h}\left(\mathbf{x}_{0}\right)\right|\right)+\bar{\beta}\left(2 i_{\varepsilon} \gamma(\|\mathbf{d}\|)+2 \varepsilon\right)+\gamma(\|\mathbf{d}\|), \\
\left|\mathbf{y}\left(t_{i+1}\right)\right| \leq \rho_{i} \ldots \rho_{0}\left|\mathbf{h}\left(\mathbf{x}_{0}\right)\right|+\left(i_{\varepsilon}+1\right) \gamma(\|\mathbf{d}\|)+\varepsilon, \quad i \geq 0 .
\end{gathered}
$$

Since $\varepsilon$ can be chosen arbitrary and due to the fact that $\gamma\left(\|\mathbf{d}\|_{[T,+\infty)}\right) \rightarrow 0$ with $T \rightarrow+\infty$, the last inequality implies that the output $\mathbf{y}(t)$ converges to zero asymptotically.
As a corollary of Theorem 3 we see exponentially stable SIIOS systems family (1) with dwell-time switching do not yield an exponentially SIIOS switched system (2) (opposite to the case of exponentially stable systems switching) and stability of the switched system follows only for integrally bounded inputs.

\section{EXAMPLE}

Consider the following family of systems for $n=1$, $I=\{0,1,2\}$ and

$$
\begin{gathered}
f_{0}(x, d)=-x /\left(1+x^{2}\right)+d \quad f_{1}(x, d)=-r x^{3}+d \\
f_{2}(x, d)=-r\left(1-e^{-x}\right) /\left(1+e^{-x}\right)+d, \quad r>0 .
\end{gathered}
$$

Direct computations show, that

$$
\begin{gathered}
\beta_{0}(s, t)=\sqrt{W\left(s^{2} e^{s^{2}} e^{-2 t}\right)} ; \beta_{1}(s, t)=s / \sqrt{2 r s^{2} t+1} \\
\beta_{2}(s, t)=\ln [\varphi(s, t)+0.5 \sqrt{\varphi(s, t)(4+\varphi(s, t))}+1] \\
\varphi(s, t):=0.5\left(e^{-s}+e^{s}-2\right) e^{-r t}
\end{gathered}
$$

where $W(s)$ is Lambert function (Omega function), which is the solution of equation $W(s) e^{W(s)}=s, s \geq 0$. For all these functions $\bar{\beta}(s)=\beta_{j}(s, 0)=s, s \geq 0, j \in I$, then as it was discussed before

$$
\rho(s) \geq \max _{j \in I} \rho_{j}(s) ; \quad \rho_{j}(s)=\frac{\beta_{j}\left(s, \tau_{D}\right)}{s}, \quad j \in I .
$$

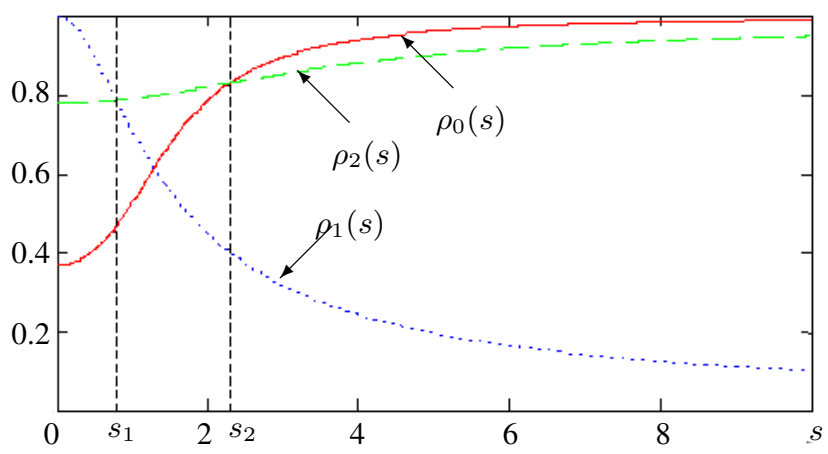

Fig. 1. Graphs of gain functions

For $r=0.5$ and $\tau_{D}=1$ functions $\rho_{j}, j \in I$ are plotted in Fig. 1. According to the latter the function $\rho(s)=$ $\max _{j \in I} \rho_{j}(s)$ can be defined as follows for $s_{1}=0.785$, $s_{2}=2.285$

$$
\rho(s)=\left\{\begin{array}{l}
\rho_{1}(s) \text { if } s \leq s_{1} \\
\rho_{0}(s) \text { if } s \geq s_{2} \\
\rho_{2}(s) \text { otherwise }
\end{array}\right.
$$

Here $\rho_{\max }=1$. Thus, according to Theorem 2 this system has bounded and converging to zero output. From Theorem 3 we conclude that these stability properties are preserved in the presence of integrally bounded inputs. The results of the system simulations are shown in Fig. 2; the state 


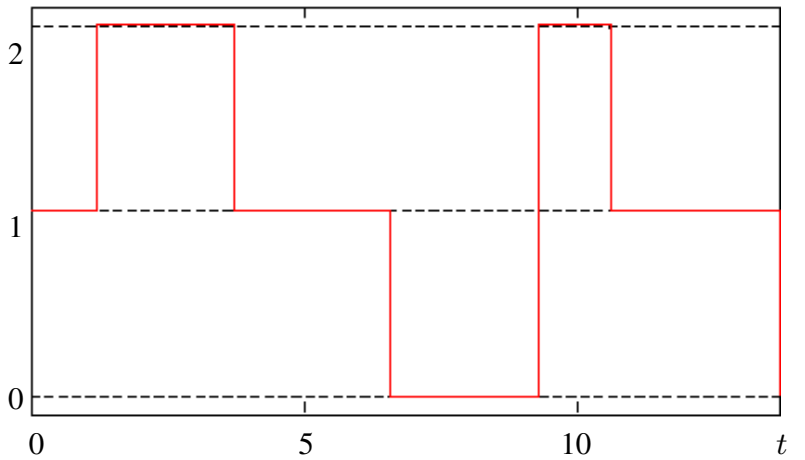

Fig. 2. Graph of switching signal $i(t)$

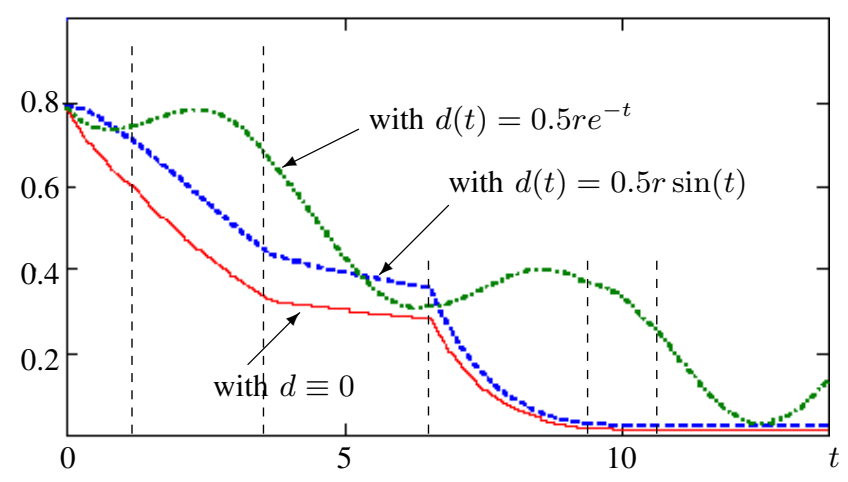

Fig. 3. System's response to different perturbations

$x(t)$ of the switched system is depicted for three cases: without disturbance $d$, with converging-to-zero disturbance $d(t)=0.5 r e^{-t}$ and finally with the bounded disturbance $d(t)=0.5 r \sin (t)$. In Fig. 3 the switching signal is shown. In this example system preserves its stability properties for the chosen bounded disturbance (the first and the last systems are SIIOS only for bounded by $r$ inputs).

\section{CONCLUSION}

The problem of output stability of switched nonlinear systems has been considered. Two solutions of the problem of non-exponentially stable systems switching are proposed. One solution is based on new type of switching between locally exponentially stable systems in (1) and the rest ones. Another solution introduces new requirement on type of decreasing under dwell-time, which allows one to take into consideration non-exponentially stable systems. Results of simulation confirm applicability of the proposed solutions.

\section{REFERENCES}

[1] Angeli D., Sontag E.D. (1999). Forward completeness, unboundedness observability, and their Lyapunov characterizations. Systems and Control Letters, 38, pp. 209-217.

[2] Efimov D.V., Panteley E., Loria A. (2008). On Input-to-
Output Stability of Switched Nonlinear Systems. Proc. 17th IFAC WC, Seoul, Korea.

[3] Hespanha J.P., D. Liberzon, Morse A.S. (2003). Hysteresisbased switching algorithms for supervisory control of uncertain systems. Automatica, 39, pp. 263-272.

[4] Hespanha J.P., Morse A.S. (1999). Stability of switched systems with average dwell-time. In Proc. 38th IEEE Conf. on Decision and Control, pp. 2655-2660.

[5] Liberzon D. (1999). ISS and integral-ISS disturbance attenuation with bounded controls. Proc. 38th IEEE Conf. on Decision and Control, pp. 2501-2506.

[6] Liberzon D. (2003). Switching in Systems and Control. Birkhäuser, Boston.

[7] Morse A.S. (1995). Control using logic-based switching. In: Trends in control (A. Isidory (Ed.)), Springer-Verlag, pp. 69-113.

[8] Morse A. S. (1996). Supervisory control of families of linear set-point controllers-part I: exact matching. IEEE Trans. Automat. Control, 41, pp. 1413-1431.

[9] Morse A. S. (1997). Supervisory control of families of linear set-point controllers-part II: robustness. IEEE Trans. Automat. Control, 42, pp. 1500-1515.

[10] C. De Persis, R. De Santis, Morse A. S. (2003). Switched nonlinear systems with state-dependent dwell-time switching logic. Systems\&Control Letters, 50, pp. 291-302.

[11] Sontag E.D. (1989). Smooth stabilization implies coprime factorization. IEEE Trans. Aut. Contr., 34, pp. 435-443.

[12] Sontag E.D., Wang Y. (1999). Notions of input to output stability. Systems and Control Letters, 38, pp. 235-248.

[13] Sontag E.D., Wang Y. (2001). Lyapunov characterizations of input to output stability. SIAM Journal on Control and Optimization, 39, pp. 226-249.

[14] Vu L., Chatterjee D., Liberzon D. (2005). ISS of Switched Systems and Applications to Switching Adaptive Control. In Proc. 44th IEEE Conf. on Decision and Control, pp. $120-125$.

[15] Xie W., Wen C., Li Z. (2001). Input-to-state stabilization of switched nonlinear systems. IEEE Trans. Automat. Control, 46, pp. 1111-1116. 